\title{
An anisotropic quasilinear problem with perturbations
}

Jie Rui ${ }^{1,2}$ and Jianguo $\mathrm{Si}^{1 *}$

"Correspondence: rjhygl@163.com 'School of Mathematics, Shandong University, Jinan, Shandong 250100 P.R. China

Full list of author information is available at the end of the article

\begin{abstract}
This work focuses on proving the existence and uniqueness of strong solutions of perturbed anisotropic total variation flow with the Neumann boundary condition when the initial data is an $L^{2}(\Omega)$ function.
\end{abstract}

MSC: 35K65; 35K55

Keywords: anisotropic total variation flow; semigroups; strong solution

\section{Introduction}

Problems of general anisotropic total variation flow arise in a number of areas of science. The parabolic equations represent what Giga et al. called a very singular diffusivity (see [1]) and are a natural generalization of the total variation flow in the presence of an anisotropy. In the isotropic case, the equation $u_{t}=\operatorname{div}\left(\partial_{\xi} f(D u)\right)$ becomes $u_{t}=\operatorname{div}\left(\frac{D u}{|D u|}\right)$ when the Lagrangian $f: \mathbb{R}^{N} \rightarrow[0,+\infty]$ is given by $f(\xi)=\|\xi\|$, where $\|\xi\|$ is the usual $l^{2}$-norm; i.e., $\|\xi\|:=\left(\sum_{i=1}^{N} \xi_{i}^{2}\right)^{\frac{1}{2}}$. Let us recall that this PDE appears when one uses the steepest decent method to minimize the total variation. This method was introduced by Rudin and Osher (see $[2,3])$ in the context of image denoising and reconstruction. In the last years, its applications have been studied by many authors (see [4-7]).

Let $\Omega$ be an open bounded subset in $\mathbb{R}^{N}, N \geq 2$, with boundary $\partial \Omega$ of class $C^{1}$. In this paper, we are interested in the problem

$$
\begin{cases}\frac{\partial u}{\partial t}=\operatorname{div}\left(\partial_{\xi} f(x, D u)\right)-h(x, u) & \text { in }[0, T] \times \Omega, \\ \frac{\partial u}{\partial \eta}=0 & \text { on }[0, T] \times \partial \Omega, \\ u(0)=u_{0} & \text { in } \Omega,\end{cases}
$$

where $u_{0} \in L^{2}(\Omega), f(x, \xi)$ is a 1-homogeneous convex function with linear growth as $\|\xi\| \rightarrow$ $\infty, \frac{\partial}{\partial \eta}$ is the Neumann boundary operator associated to $\partial_{\xi} f(x, \xi)$, i.e., $\frac{\partial u}{\partial \eta}:=\partial_{\xi} f(x, \xi) \cdot v$ with $v$ the unit outward normal on $\partial \Omega$, and the function $h(x, u): \Omega \times \mathbb{R} \rightarrow \mathbb{R}$ satisfies the following assumptions, which we shall refer to collectively as (M):

$\left(\mathrm{M}_{1}\right)$ For almost all $x \in \Omega, r \rightarrow h(x, r)$ is continuous nondecreasing, and $h(x, 0)=0$;

$\left(\mathrm{M}_{2}\right)$ For every $r \in \mathbb{R}, x \rightarrow h(x, r)$ is in $L^{2}(\Omega)$.

As argued in [8], the choice of Neumann boundary conditions is a natural choice in image processing. It corresponds to the reflection of the picture across the boundary and has the advantage of not imposing any value on boundary and not creating edges

(c) 2013 Rui and Si; licensee Springer. This is an Open Access article distributed under the terms of the Creative Commons Attribution License (http://creativecommons.org/licenses/by/2.0), which permits unrestricted use, distribution, and reproduction in any medium, provided the original work is properly cited. 
on it. For instance, in [9], Andreu, Caselles and Mazón considered the elliptic problem $u-\operatorname{div} \mathbf{a}(u, D u)=g$ with Neumann boundary conditions. In [7], Andreu et al. obtained the existence and uniqueness of entropy solutions of quasilinear parabolic equation with the Neumann boundary, i.e.,

$$
\begin{cases}\frac{\partial u}{\partial t}=\operatorname{div} \mathbf{a}(u, D u) & \text { in }(0, T) \times \Omega, \\ \frac{\partial u}{\partial \eta}=0 & \text { on }(0, T) \times \partial \Omega, \\ u(0)=u_{0} & \text { in } \Omega,\end{cases}
$$

where $u(0)=u_{0} \in L^{1}(\Omega), \mathbf{a}(z, \xi)=\nabla_{\xi} f(z, \xi)$, and $f \in C^{1}$ satisfies some additional assumptions. Our problem is closely related to motion under anisotropic mean curvature flow (see [10]) when $h(x, u) \equiv 0$. If we take the $f^{0}$-distance to give a set $E$ as an initial condition $\left(f^{0}\right.$ being the polar function of $f$ ), then each sublevel set of the anisotropic mean curvature motion behaves instantaneously as the solution of Cauchy problem (1.1) where $\Omega=\mathbb{R}^{N}$. Recently Moll [11] proved the existence and uniqueness of the solutions of Dirichlet problem (1.1) with $h(x, u) \equiv 0$. As we all know, it is possible that the solution of (1.1) will blow up with perturbations. Therefore, in this paper, we extend the problem introduced in Moll [11] and obtain the existence and uniqueness of strong solutions of (1.1) when perturbation term satisfies assumption $(\mathrm{M})$.

This paper is organized as follows. In Section 2 we recall some notions and basic facts. In Section 3 we define the notion of a strong solution for the Neumann problem of (1.1), and give the basic results in this paper. In Section 4 we prove the existence and uniqueness of solutions of an auxiliary equation, i.e.,

$$
\begin{cases}\frac{\partial u}{\partial t}=\operatorname{div}\left(\partial_{\xi} f(x, D u)\right) & \text { in }[0, T] \times \Omega, \\ \frac{\partial u}{\partial \eta}=0 & \text { on }[0, T] \times \partial \Omega, \\ u(0)=u_{0} & \text { in } \Omega,\end{cases}
$$

and for some $h(x, u)$ we obtain the existence and uniqueness of a strong solution of problem (1.1).

\section{Preliminaries}

To make precise our notions, let us recall some preliminary facts.

Given $u \in \mathrm{BV}(\Omega)$, $D u$ decomposes into absolutely continuous and singular parts $D u=$ $\nabla u L^{N} L_{\Omega}+D^{s} u$, where $\nabla u$ denotes the Radon-Nikodým derivative with respect to the Lebesgue measure and $D^{s} u$ is its singular part. There is also the polar decomposition $D^{s} u=\overrightarrow{D^{s} u}\left|D^{s} u\right|$, where $\left|D^{s} u\right|$ is the total variation measure of $D^{s} u$. For further information concerning functions of bounded variation, we refer to [12].

By $L_{w}^{1}([0, T] ; \mathrm{BV}(\Omega))$ we denote the space of weakly measurable functions $w:[0, T] \rightarrow$ $\mathrm{BV}(\Omega)$ (i.e., $t \in[0, T] \rightarrow\langle w(t), \phi\rangle$ is measurable for every $\left.\phi \in \mathrm{BV}(\Omega)^{*}\right)$ such that $\int_{0}^{T}\|w(t)\|<$ $+\infty$. Observe that since $\operatorname{BV}(\Omega)$ has separable predual, it follows easily that the map $t \in[0, T] \rightarrow\|w(t)\|$ is measurable.

We shall need several results from [13] in order to give sense to the integrals of bounded vector fields with divergence in $L^{p}(\Omega)$. Let $p \geq 1$ and $p^{\prime} \geq 1$ be such that $\frac{1}{p}+\frac{1}{p^{\prime}}=1$. Following [13], let

$$
X_{p}(\Omega)=\left\{z \in L^{\infty}\left(\Omega, \mathbb{R}^{N}\right): \operatorname{div}(z) \in L^{p}(\Omega)\right\} .
$$


If $z \in X_{p}(\Omega)$ and $u \in \operatorname{BV}(\Omega) \cap L^{p}(\Omega)$, the functional $(z, D u): C_{0}^{\infty}(\Omega) \rightarrow \mathbb{R}$ is defined by the formula

$$
\langle(z, D u), \varphi\rangle=-\int_{\Omega} u \varphi \operatorname{div}(z) d x-\int_{\Omega} u z \cdot \nabla \varphi d x .
$$

Then $(z, D u)$ is a Radon measure in $\Omega, \int_{\Omega}(z, D w)=\int_{\Omega} z \cdot \nabla w d x$ for all $w \in W^{1,1}(\Omega) \cap$ $L^{\infty}(\Omega)$, and $(z, D w)$ is absolutely continuous with respect to $\|D w\|$ with the RadonNikodým derivative $\theta(z, D w, x)$ which is a $\|D w\|$ measurable function from $\Omega$ to $\mathbb{R}$ such that $\int_{B}(z, D w)=\int_{B} \theta(z, D w, x)\|D w\|$ for any Borel set $B \subseteq \Omega$. We also have that $\|\theta(z, D w, \cdot)\|_{L^{\infty}(\Omega,\|D w\|)} \leq\|z\|_{L^{\infty}\left(\Omega, \mathbb{R}^{N}\right)}$.

In [13], a weak trace on $\partial \Omega$ of the normal component of $z \in X_{p}(\Omega)$ is defined. Concretely, it is proved that there exists a linear operator $\gamma: X_{p}(\Omega) \rightarrow L^{\infty}(\partial \Omega)$ such that $\|\gamma(z)\|_{\infty} \leq$ $\|z\|_{\infty}$ and $\gamma(z)(x)=z(x) \cdot v(x)$ for all $x \in \partial \Omega$ if $z \in C^{1}\left(\bar{\Omega}, \mathbb{R}^{N}\right)$.

Next, let us introduce the concept of generalized total variation of a BV function with respect to a Finsler metric [14]. Let $f: \bar{\Omega} \times \mathbb{R}^{N} \rightarrow[0, \infty]$ be a Borel function not identically $+\infty$. The function $f$ will be called convex if for any $x \in \Omega$, the function $f(x, \cdot)$ is convex on $\mathbb{R}^{N}$. We shall say that $f$ is lower semicontinuous (in short l.s.c.) if $f(x, \cdot)$ is lower semicontinuous for any $x \in \Omega$. The function will be called positively homogeneous of degree 1 (in short 1-homogeneous) if it satisfies the following property:

$$
f(x, t \xi)=|t| f(x, \xi), \quad \forall x \in \Omega, \forall \xi \in \mathbb{R}^{N}, \forall t \in \mathbb{R}
$$

$f$ is a sublinear growth if there exists a positive constant $0<C_{0}<+\infty$ such that

$$
0 \leq f(x, \xi) \leq C_{0}\|\xi\|, \quad \forall x \in \Omega, \forall \xi \in \mathbb{R}^{N}
$$

Let us recall that $f: \bar{\Omega} \times \mathbb{R}^{N} \rightarrow[0, \infty]$ is a Finsler metric if it is a Borel function and it satisfies (2.1) and (2.2). If $f$ satisfies (2.1), then the dual function $f^{*}: \Omega \times \mathbb{R}^{N} \rightarrow[0,+\infty]$ is defined by $f^{*}(x, \xi)=\sup \left\{\left(\xi, \xi^{*}\right): \xi^{*} \in \mathbb{R}^{N}, f\left(x, \xi^{*}\right) \leq 1\right\}$. It is easy to verify that $f^{*}$ is convex, l.s.c. and satisfies (2.1). Then, if we adopt the following conventions: for any $a \in[0,+\infty]$, we set $\frac{a}{+\infty}=0 ; \frac{a}{0}=+\infty$ if $a \neq 0$ and $\frac{0}{0}=0$, we get

$$
f^{*}(x, \xi)=\sup \left\{\frac{\left(\xi, \xi^{*}\right)}{f\left(x, \xi^{*}\right)}: \xi^{*} \in \mathbb{R}^{N}\right\}, \quad \forall x \in \Omega, \forall \xi \in \mathbb{R}^{N}
$$

We say that $f$ is coercive if there exists a positive constant $0<C_{1}<+\infty$ such that

$$
f(x, \xi) \geq C_{1}\|\xi\|, \quad \forall x \in \Omega, \forall \xi \in \mathbb{R}^{N} .
$$

It is easy to see that $f$ is convex and has a sublinear growth, then $f(x, \cdot)$ is continuous for any $x \in \Omega$.

We introduce the classes of vector fields

$$
\begin{aligned}
& X_{c}^{p}(\Omega):=\left\{z \in X_{p}(\Omega): \operatorname{supp}(z) \text { is compact in } \Omega\right\}, \\
& \mathcal{H}_{f}(\Omega):=\left\{z \in X_{c}^{p}(\Omega): f^{\prime \prime}(x, z(x)) \leq 1 \text { for a.e. } x \in \Omega\right\}
\end{aligned}
$$


and

$$
\mathcal{M}_{f}^{p}(\Omega):=\left\{z \in X_{p}(\Omega): f^{*}(x, z(x)) \leq 1 \text { for a.e. } x \in \Omega\right\}
$$

Let $u \in \mathrm{BV}(\Omega)$, the generalized total variation of $u$ with respect to $f$ in $\Omega$ is defined by

$$
\int_{\Omega}|D u|_{f}:=\sup \left\{\int_{\Omega}(z, D u): z \in \mathcal{H}_{f}(\Omega)\right\}=\sup \left\{\int_{\Omega} u \operatorname{div}(z) d x: z \in \mathcal{H}_{f}(\Omega)\right\} .
$$

As a direct consequence of the definition, we have that the generalized total variation of $u$ with respect to $f$ in $\Omega$ is $L^{1}(\Omega)$-lower semicontinuous in $\Omega$.

Now, we introduce the relaxed functional, which plays a basic role in proving the existence and uniqueness of the problem.

We define the functional $G[f]: L^{1}(\Omega) \rightarrow[0,+\infty]$ by

$$
G[f](u):= \begin{cases}\int_{\Omega} f(x, \nabla u(x)) d x & \text { if } u \in W^{1,1}(\Omega) \\ +\infty, & \text { otherwise }\end{cases}
$$

We denote by $\mathcal{G}[f]$ the relaxed functional of $G[f]$; i.e.,

$$
\mathcal{G}[f](u):=\inf _{\left\{u_{n}\right\}}\left\{\liminf _{n \rightarrow \infty} G[f]\left(u_{n}\right): u_{n} \in W^{1,1}(\Omega), u_{n} \rightarrow u \in L^{1}(\Omega)\right\}
$$

In [14], Amer and Belletini obtained the following result:

$$
\int_{\Omega}|D u|_{f}=\mathcal{G}\left[f^{* * *}\right](u)=\mathcal{G}[f](u), \quad \forall u \in \operatorname{BV}(\Omega) .
$$

Moreover, in [11], Moll proved the representation result:

$$
\int_{\Omega}|D u|_{f}=\int_{\Omega}[\mathcal{R}(f)]\left(x, v^{u}\right)|D u|, \quad \forall u \in \mathrm{BV}(\Omega),
$$

where $[\mathcal{R}(f)]: \Omega \times \mathbb{R}^{N} \rightarrow[0,+\infty]$ is a representative of the equivalence class of homogeneous integrands $h_{D}$ associated to sets $D \subseteq K=\mathcal{M}_{f}^{p}(\Omega)$ which are countable and sequentially weakly"-dense in $\mathcal{M}_{f}^{p}(\Omega)$, and

$$
\int_{\Omega}[\mathcal{R}(f)]\left(x, v^{u}\right)|D u|=\int_{\Omega} j(x, \nabla u(x)) d x+\int_{\Omega}[\mathcal{R}(f)]\left(x, v^{u}\right)\left|D^{s} u\right|
$$

for all $u \in \mathrm{BV}(\Omega)$, where $j(x, \xi)=\sup \left\{z(x) \cdot \xi: z \in \mathcal{M}_{f}^{p}(\Omega)\right\}$. The following useful inequality holds:

$$
j(x, \xi) \leq \sup \left\{\xi^{*} \cdot \xi: \xi^{*} \in \mathbb{R}^{N}, f^{*}\left(x, \xi^{*}\right) \leq 1\right\}=f(x, \xi), \quad \forall(x, \xi) \in \Omega \times \mathbb{R}^{N}
$$

The equality holds if and only if the functional $G[f]$ defined by $(2.5)$ is $L^{1}(\Omega)$-lower semicontinuous on $W^{1,1}(\Omega)$. By the inequality (2.9), we have the measure $|D u|_{f}$ as follows:

$$
|D u|_{f}(B)=\int_{\Omega \cap B}|D u|_{f}=\int_{\Omega \cap B} j(x, \nabla u(x)) d x+\int_{\Omega \cap B}[\mathcal{R}(f)]\left(x, v^{u}\right)\left|D^{s} u\right|
$$

for every Borel set $B \subseteq \mathbb{R}^{N}$. 
In this paper, we assume that $f: \Omega \times \mathbb{R}^{N} \rightarrow[0,+\infty]$ is a convex homogeneous integrand, i.e., for some constants $C_{0}, C_{1}>0$

$$
C_{1}\|\xi\| \leq f(x, \xi) \leq C_{0}\|\xi\|, \quad \forall(x, \xi) \in \Omega \times \mathbb{R}^{N}
$$

Let us define the functional $F(u): L^{1}(\Omega) \rightarrow[0,+\infty]$ by the formula

$$
F(u):= \begin{cases}\int_{\Omega} f(x, \nabla u(x)) d x, & u \in W^{1,1}(\Omega) \text { and } \frac{\partial u}{\partial \eta}=0 \text { on } \partial \Omega, \\ +\infty, & \text { otherwise. }\end{cases}
$$

By $\frac{\partial u}{\partial \eta}=0$ on $\partial \Omega$, and Theorem 4 in [11], it is easy to obtain that the functional $\mathcal{F}$ : $\mathrm{BV}(\Omega) \rightarrow[0,+\infty]$ is the relaxed functional of $F$ defined by

$$
\mathcal{F}(u):=\int_{\Omega}|D u|_{f}
$$

\section{Strong solutions and main results}

In this section we give the main concepts and results of Neumann problems (1.3) and (1.1).

Definition 3.1 A function $u \in C\left([0, T] ; L^{2}(\Omega)\right)$ is a strong solution of (1.3) if $u \in W_{\text {loc }}^{1,2}([0$, $\left.T] ; L^{2}(\Omega)\right) \cap L_{w}^{1}([0, T] ; \mathrm{BV}(\Omega))$, there exists $z \in L^{\infty}\left([0, T] \times \Omega ; \mathbb{R}^{N}\right)$ with $f^{*}(x, z(t, x)) \leq 1$ a.e. in $\mathbb{R}^{N}$ and a.e. $t>0$, such that

$$
u_{t}=\operatorname{div}(z) \quad \text { in } \mathcal{D}^{\prime}([0, T] \times \Omega)
$$

and a.e. $t>0$ it holds

$$
\begin{aligned}
& \int_{\Omega}(z(t), D u(t))=\int_{\Omega}|D u(t)|_{f}, \\
& {[z(t), v]=0, \quad \mathcal{H}^{N-1} \text {-a.e. on } \partial \Omega .}
\end{aligned}
$$

Next we give the main definition in this paper that is the strong solution of problem (1.1).

Definition 3.2 A function $u \in C\left([0, T] ; L^{2}(\Omega)\right)$ is a strong solution of (1.1) if $u \in W_{\text {loc }}^{1,2}([0$, $\left.T] ; L^{2}(\Omega)\right) \cap L_{w}^{1}([0, T] ; \mathrm{BV}(\Omega))$, there exists $z \in L^{\infty}\left([0, T] \times \Omega ; \mathbb{R}^{N}\right)$ with $f^{*}(x, z(t, x)) \leq 1$ a.e. in $\mathbb{R}^{N}$ and $u_{t}=\operatorname{div}(z)-h(x, u)$ in $\mathcal{D}^{\prime}([0, T] \times \Omega)$ a.e. $t>0$ such that

$$
\int_{\Omega}(u(t)-w) u_{t} \leq \int_{\Omega}(z(t), D w)-\int_{\Omega}|D u(t)|_{f}+\int_{\Omega}(w-u(t)) h(x, u(t))
$$

for every $w \in W^{1,1}(\Omega) \cap L^{2}(\Omega)$ and a.e. on $[0, T]$.

The main results of this paper are the following.

Theorem 3.3 Let $u_{0} \in L^{2}(\Omega)$. Assume that $f$ satisfies (2.11), then there exists a unique strong solution of $(1.3)$ in $[0, T] \times \Omega$ for every $T>0$ such that $u(0)=u_{0}$. Moreover, if $\widetilde{u(t)}$, $u(t)$ are the strong solutions of (1.3) corresponding to initial data $\widetilde{u_{0}}, u_{0}$, respectively, then

$$
\left\|(\widetilde{u(t)}-u(t))^{+}\right\|_{2} \leq\left\|\left(\widetilde{u_{0}}-u_{0}\right)^{+}\right\|_{2} \text { for any } t>0 .
$$


Theorem 3.4 Let $u_{0} \in L^{2}(\Omega)$. Assume that $f$ satisfies $(2.11)$ and $h(x, u)$ satisfies $(\mathrm{M})$, then there exists a unique strong solution of $(1.1)$ in $[0, T] \times \Omega$ for every $T>0$ such that $u(0)=u_{0}$. Moreover, if $\hat{u}(t)_{1}, \hat{u}(t)_{2}$ are the strong solutions of $(1.1)$ corresponding to initial data $\hat{u}_{01}, \hat{u}_{02}$, respectively, then

$$
\left\|\left(\hat{u}(t)_{1}-\hat{u}(t)_{2}\right)^{+}\right\|_{2} \leq\left\|\left(\hat{u}_{01}-\hat{u}_{02}\right)^{+}\right\|_{2} \text { for any } t>0 .
$$

\section{Proof of the main results}

In this section we prove Theorem 3.3 by using the techniques of completely accretive operators [15] and Crandall-Liggett's semigroup generation theorem [16].

Let us recall the notion of completely accretive operators introduced in [15]. Let $\mathcal{M}(\Omega)$ be the space of measurable functions in $\Omega$. Given $u, v \in \mathcal{M}(\Omega)$, we shall write that $u \ll v$ if and only if $\int_{\Omega} j(u) d x \leq \int_{\Omega} j(v) d x$ for all $j \in J_{0}$, where $J_{0}=\{j: \mathbb{R} \rightarrow$ $[0, \infty]$, convex, l.s.c., $j(0)=0\}$. Let $A$ be an operator (possibly multivalued) in $\mathcal{M}(\Omega)$, i.e., $A \subseteq \mathcal{M}(\Omega) \times \mathcal{M}(\Omega)$. We shall say that $A$ is completely accretive if

$$
u-\tilde{u} \ll u-\tilde{u}+\lambda(v-\tilde{v}) \quad \text { for all } \lambda>0 \text { and all }(u, v),(\tilde{u}, \tilde{v}) \in A \text {. }
$$

Let $P_{0}=\left\{p \in C^{\infty}(\mathbb{R}): 0 \leq p^{\prime} \leq 1, \operatorname{supp}\left(p^{\prime}\right)\right.$ be compact and $\left.0 \notin \operatorname{supp}\left(p^{\prime}\right)\right\}$. If $A \subseteq L^{2}(\Omega) \times$ $L^{2}(\Omega)$, then $A$ is completely accretive if and only if $\int_{\Omega} p(u-\tilde{u})(v-\tilde{v}) \geq 0$ for any $p \in P_{0}$, $(u, v),(\tilde{u}, \tilde{v}) \in A$. A completely accretive operator in $L^{2}(\Omega)$ is said to be $m$-completely accretive if $R(I+\lambda A)=L^{2}(\Omega)$ for any $\lambda>0$. In that case, by Crandall-Liggett's theorem, $A$ generates a contraction semigroup denoted by $\{S(t)\}_{t>0}$ in $L^{2}(\Omega)$, which is given by the exponential formula

$$
S(t) u_{0}=e^{-t A} u_{0}=\lim _{n \rightarrow \infty}\left(I+\frac{t}{n} A\right)^{-n} u_{0} \quad \text { for any } u_{0} \in L^{2}(\Omega) .
$$

Let us write $u(t)=e^{-t A} u_{0}=S(t) u_{0}$, then $u \in C\left([0, T], L^{2}(\Omega)\right)$ for any $T>0$, and it is a mild solution (a solution in the sense of semigroups [15]) of

$$
\frac{d u}{d t}+A u \ni 0
$$

such that $u(0)=u_{0}$.

We shall use a stronger notion of the solution of (4.1). We say that $v \in C\left([0, T], L^{2}(\Omega)\right)$ is a strong solution of $(4.1)$ on $[0, T]$ if $v \in W_{\text {loc }}^{1,2}\left((0, T), L^{2}(\Omega)\right)$ and $v^{\prime}(t)+A v(t) \ni 0$ for almost all $t \in(0, T)$. If $u_{0} \in \mathcal{D}(A)=\left\{\bar{u} \in L^{2}(\Omega):(\bar{u}, \bar{v}) \in A\right.$ for some $\left.\bar{v} \in L^{2}(\Omega)\right\}$ (the domain of $A$ ) and $A$ is m-completely accretive, then $u \in W_{\mathrm{loc}}^{1,2}\left((0, T), L^{2}(\Omega)\right)$ and $u(t)$ is a strong solution of (4.1) on $(0, T)$ for all $T>0$.

To obtain the solution of problem (1.1), we need the result of problem (1.3). Thus, at first, we will prove the existence and uniqueness of a strong solution of problem (1.3). Let us introduce the following operator $\mathcal{A}$ in $L^{2}(\Omega)$ associated to problem (1.3).

$(u, v) \in \mathcal{A}$ if and only if $u \in \mathrm{BV}(\Omega) \cap L^{2}(\Omega), v \in L^{2}(\Omega)$ and there exists $z \in \mathcal{M}_{f}^{2}(\Omega), v=$ $-\operatorname{div}(z)$ in $\mathcal{D}^{\prime}(\Omega)$ such that

$$
\int_{\Omega}(w-u) v d x \leq \int_{\Omega} z \cdot \nabla w d x-\int_{\Omega}|D u|_{f}
$$

for all $w \in W^{1,1}(\Omega) \cap L^{2}(\Omega)$. 
Proposition 4.1 The operator $\mathcal{A}$ is m-completely accretive with dense domain. For any $u_{0} \in L^{2}(\Omega)$, the semigroup solution $u(t)=e^{-t \mathcal{A}} u_{0}$ is a mild solution of

$$
\left\{\begin{array}{l}
\frac{d u}{d t}+\mathcal{A} u \ni 0 \\
u(0)=u_{0}
\end{array}\right.
$$

To prove Proposition 4.1, we need to prove the operator $\mathcal{A}$ has some characterization, satisfies the range condition and has dense domain in $L^{2}(\Omega)$.

By the results of Section 2, the relaxed functional $\mathcal{F}$ is convex and lower semicontinuous. Therefore, the subdifferential $\partial \mathcal{F}$ of $\mathcal{F}$ is a maximal monotone operator in $L^{2}(\Omega)$, and consequently, if $\{T(t)\}_{t>0}$ is the semigroup solution in $L^{2}(\Omega)$ generated by $\partial \mathcal{F}, u(t)=T(t) u_{0}$ is a strong solution of the problem (see [15])

$$
\begin{cases}\frac{d u}{d t}+\partial \mathcal{F} u(t) \ni 0, & t \in[0,+\infty], \\ u(0)=u_{0}, & u_{0} \in L^{2}(\Omega) .\end{cases}
$$

Recall that the operator $\partial \mathcal{F}$ is defined by $(u, v) \in \partial \mathcal{F}$ if and only if $u, v \in L^{2}(\Omega)$ and $\mathcal{F}(w)-$ $\mathcal{F}(u) \geq \int_{\Omega} v(w-u) d x, \forall w \in L^{2}(\Omega)$.

To prove the existence and uniqueness of a strong solution of problem (1.3), we also need the next proposition.

Proposition 4.2 The operator $\partial \mathcal{F}$ has dense domain in $L^{2}(\Omega)$ and $\partial \mathcal{F}=\mathcal{A}$.

The following lemmas will be used to prove Proposition 4.1 and Proposition 4.2.

Lemma 4.3 We have the following characterization of the operator $\mathcal{A},(u, v) \in \mathcal{A}$ if and only if $u \in \mathrm{BV}(\Omega) \cap L^{2}(\Omega), v \in L^{2}(\Omega)$ and there exists $z \in \mathcal{M}_{f}^{2}(\Omega), v=-\operatorname{div}(z)$ in $\mathcal{D}^{\prime}(\Omega)$ such that

$$
\int_{\Omega}(w-u) v d x \leq \int_{\Omega}(z, D w)-\int_{\Omega}|D u|_{f}
$$

for all $w \in \mathrm{BV}(\Omega) \cap L^{2}(\Omega)$. Moreover, we have that

(i) $\int_{\Omega}(z, D u)=\int_{\Omega}|D u|_{f}$,

(ii) $\int_{\Omega} v u=\int_{\Omega}|D u|_{f}$,

(iii) $\int_{\Omega} w v=\int_{\Omega}(z, D w)$ for all $w \in \mathrm{BV}(\Omega) \cap L^{2}(\Omega)$

Proof We denote the operator by $\mathcal{B}$ defined in the statement of the lemma. Since $\int_{\Omega} z$. $\nabla w=\int_{\Omega}(z, D w)$ when $w \in W^{1,1}(\Omega) \cap L^{2}(\Omega)$, we have $\mathcal{B} \subseteq \mathcal{A}$. Let $u \in \operatorname{BV}(\Omega) \cap L^{2}(\Omega), v \in$ $L^{2}(\Omega)$ and there exists $z \in \mathcal{M}_{f}^{2}(\Omega), v=-\operatorname{div}(z)$ in $\mathcal{D}^{\prime}(\Omega)$ and (4.2). Let $w \in \operatorname{BV}(\Omega) \cap L^{2}(\Omega)$, applying results from [13], we have that there exists a sequence $w_{n} \in W^{1,1}(\Omega) \cap L^{\infty}(\Omega)$ such that $w_{n} \rightarrow w$ in $L^{1}(\Omega), \int_{\Omega}\left|\nabla w_{n}\right| \rightarrow \int_{\Omega}|D w|$ and $\int_{\Omega} z \cdot \nabla w_{n}=\int_{\Omega}\left(z, D w_{n}\right) \rightarrow \int_{\Omega}(z, D w)$. Using $w_{n}$ as a test function in (4.2) and letting $n \rightarrow \infty$, we obtain (4.5), then we conclude that $\mathcal{A} \subseteq \mathcal{B}$, therefore $\mathcal{A}=\mathcal{B}$.

Letting $w=u$ in (4.5), we get

$$
\int_{\Omega}(z, D u) \geq \int_{\Omega}|D u|_{f}
$$

Moreover, using $\int_{\Omega}|D u|_{f}:=\sup \left\{\int_{\Omega}(z, D u): z \in \mathcal{H}_{f}(\Omega)\right\}$, we obtain (i). 
We take $w=0$ in (4.5) to obtain $\int_{\Omega} u v d x \geq \int_{\Omega}|D u|_{f}$. Using $w=2 u$ in (4.5) and (i), we get

$$
\int_{\Omega} u v d x \leq 2 \int_{\Omega}(z, D u)-\int_{\Omega}|D u|_{f}=\int_{\Omega}|D u|_{f} .
$$

Thus, (ii) holds.

Using (ii) in (4.5) we have

$$
\int_{\Omega} w v d x \leq \int_{\Omega}(z, D w), \quad \forall w \in \operatorname{BV}(\Omega) \cap L^{2}(\Omega) .
$$

Since the same inequality holds for $-w \in \operatorname{BV}(\Omega) \cap L^{2}(\Omega)$, (iii) is obtained.

We consider the following possibly multi-valued functions: $A(x, \xi):=\partial_{\xi} f(x, \xi)$ and $B(x, \xi):=\partial_{\xi} j(x, \xi)$. By the convexity of $f$, it follows that $A$ is a monotone function satisfying

$$
C_{0} \leq\|\delta\| \leq C_{1}, \quad \forall \delta \in A(x, \xi)
$$

For each $x \in \Omega$, we consider the Moreau-Yosida approximation to $f(x): \mathbb{R} \rightarrow[0,+\infty]$ defined by

$$
f_{\lambda}(x)(\xi):=\min _{\zeta \in \mathbb{R}^{n}}\left\{\frac{1}{2 \lambda}\|\zeta-\xi\|^{2}+f(x)(\zeta)\right\}
$$

and the Yosida approximation of the multi-valued function $A(x)$ is defined as

$$
A_{\lambda}(x)(\xi)=\frac{I-(I+\lambda A(x))^{-1}}{\lambda} .
$$

We have that $\xi \mapsto f_{\lambda}(x)(\xi)$ is a convex Fréchet differentiable function (see [17]) such that $f_{\lambda}(x) \rightarrow f(x)$ pointwise and a.e. in $\Omega$ when $\lambda \rightarrow 0$ and $A_{\lambda}(x)(\xi)=\nabla f_{\lambda}(x)(\xi)$. Moreover, when $\zeta=J_{\lambda}(x)(\xi)=(I+\lambda A(x))^{-1} \xi$, we get the minimum in (4.6). In [11], Moll gave the following estimate:

$$
\begin{aligned}
& C_{1}\|\xi\| \geq f(x, \xi) \geq f_{\lambda}(x)(\xi) \geq \begin{cases}\frac{C_{0}}{2}\|\xi\| & \text { if }\|\xi\| \geq C_{0} \lambda, \\
\frac{1}{2 \lambda}\|\xi\|^{2} & \text { if }\|\xi\|<C_{0} \lambda,\end{cases} \\
& A_{\lambda}(x)(\xi) \cdot \xi \geq f_{\lambda}(x)(\xi) \text { and } \quad A_{\lambda}(x)(\xi) \cdot \xi \leq C_{1}\|\xi\| .
\end{aligned}
$$

We consider the operator $A_{n}(x, \xi)=A_{\frac{1}{n}}(x, \xi)+\frac{1}{n} \xi$ in $L^{2}(\Omega)$ to prove Proposition 4.1. Let $W_{p}^{1,2}(\Omega):=\left\{w \in W^{1,2}(\Omega): \frac{\partial w}{\partial \eta}=0\right.$ on $\left.\partial \Omega\right\}$, we define $(u, v) \in A_{n}$ if and only if $u \in W_{p}^{1,2}(\Omega) \cap$ $L^{\infty}(\Omega), v \in L^{1}(\Omega)$ and

$$
\int_{\Omega}(w-u) v d x \leq \int_{\Omega} A_{n}(x)(\nabla u) \cdot \nabla(w-u) d x
$$

for all $w \in W_{p}^{1,2}(\Omega) \cap L^{\infty}(\Omega)$.

The operator $A_{n}$ satisfies the classical Leray-Lions assumption [18]. Hence, for every $n \in \mathbb{N}$, the operator $A_{n}$ satisfies $L^{\infty}(\Omega) \subset R\left(I+A_{n}\right)$.

Moreover, we need the following characterization of the operator $A_{n}$. 
Lemma 4.4 For every $n \in \mathbb{N}$, the operator $A_{n}$ is completely accretive in $L^{2}(\Omega)$.

Proof Let $p \in P_{0}$ and $(u, v),(\tilde{u}, \tilde{v}) \in A_{n}$. Since $(u, v) \in A_{n}$, taking $w=u-p(u-\tilde{u})$ as a test function in (4.10), we get

$$
\int_{\Omega} p(u-\tilde{u}) v d x \geq \int_{\Omega} A_{n}(x)(\nabla u) \cdot \nabla p(u-\tilde{u}) d x .
$$

Similarly, $(\tilde{u}, \tilde{v}) \in A_{n}$, we take $w=\tilde{u}+p(u-\tilde{u})$ as a test function in (4.10) and obtain

$$
-\int_{\Omega} p(u-\tilde{u}) \tilde{v} d x \geq-\int_{\Omega} A_{n}(x)(\nabla \tilde{u}) \cdot \nabla p(u-\tilde{u}) d x
$$

Using (4.11) + (4.12), we may write that

$$
\int_{\Omega} p(u-\tilde{u})(v-\tilde{v}) d x \geq \int_{\Omega} A_{n}(x)(\nabla u-\nabla \tilde{u}) \cdot \nabla p(u-\tilde{u}) d x .
$$

According to (4.6) and $u \in W_{p}^{1,2}(\Omega) \cap L^{\infty}(\Omega)$, we obtain that $q:=A_{n}(x)(\nabla u) \in X_{p}(\Omega)$. Moreover, by Lemma 4.3 and Theorem 2 in [4], we have that

$$
\begin{aligned}
\int_{\Omega} p(u-\tilde{u})(v-\tilde{v}) d x & \geq \int_{\Omega}(q-\tilde{q}) \cdot \nabla p(u-\tilde{u}) d x \\
& =\int_{\Omega}((q-\tilde{q}), D p(u-\tilde{u})) \\
& =\int_{\Omega} \theta((q-\tilde{q}), D p(u-\tilde{u}), x)|D p(u-\tilde{u})| \\
& \geq 0 .
\end{aligned}
$$

It follows that the operator $A_{n}$ is completely accretive in $L^{2}(\Omega)$.

Lemma 4.5 The operator $\mathcal{A}$ satisfies $L^{\infty}(\Omega) \subset R(I+\mathcal{A})$, and $D(\mathcal{A})$ is dense in $L^{2}(\Omega)$.

Proof We divide the proof into two steps.

Step 1. We first prove $L^{\infty}(\Omega) \subset R(I+\mathcal{A})$. Let $v \in L^{\infty}(\Omega)$, we shall find $u \in \operatorname{BV}(\Omega) \cap L^{2}(\Omega)$ such that $(u, v-u) \in \mathcal{A}$, i.e., there exists $z \in X_{2}(\Omega)$ such that $f^{*}(x, z(x)) \leq 1, L^{N}$-a.e. $x \in \Omega$, $v-u=-\operatorname{div}(z)$ and

$$
\int_{\Omega}(w-u)(v-u) d x \leq \int_{\Omega} z \cdot \nabla w d x-\int_{\Omega}|D u|_{f}
$$

for all $w \in W^{1,1}(\Omega) \cap L^{2}(\Omega)$.

Using (4.10) and $L^{\infty}(\Omega) \subset R\left(I+A_{n}\right)$, we have that for every $n \in \mathbb{N}$ there is $u_{n} \in W_{p}^{1,2}(\Omega) \cap$ $L^{\infty}(\Omega)$ such that $\left(u_{n} \cdot v-u_{n}\right) \in A_{n}$ and

$$
\int_{\Omega}\left(w-u_{n}\right)\left(v-u_{n}\right) d x \leq \int_{\Omega} A_{n}(x)\left(\nabla u_{n}\right) \cdot \nabla\left(w-u_{n}\right) d x
$$

for all $w \in W_{p}^{1,2}(\Omega) \cap L^{\infty}(\Omega)$. Since $A_{n}$ is completely accretive, it is obtained that

$$
\left\|u_{n}\right\|_{\infty}=\left\|\left(I+A_{n}\right)^{-1} v\right\|_{\infty} \leq\|v\|_{\infty}
$$


Now taking $w=2 u_{n}$ and $w=-u_{n}$ in (4.14), respectively, we get that

$$
\int_{\Omega} A_{n}(x)\left(\nabla u_{n}\right) \cdot \nabla u_{n} d x=\int_{\Omega} u_{n}\left(v-u_{n}\right) d x
$$

Using the estimate (4.9), (4.8) and $\Omega$ being a bounded subset in $\mathbb{R}^{N}$, we have that

$$
\int_{\Omega} A_{\frac{1}{n}}(x)\left(\nabla u_{n}\right) \cdot \nabla u_{n} d x \geq C_{0}\left\|\nabla u_{n}\right\|
$$

By (4.15), it follows that

$$
\left\|\nabla u_{n}\right\|+\frac{1}{n} \int_{\Omega}\left|\nabla u_{n}\right|^{2} d x \leq \int_{\Omega} u_{n}\left(v-u_{n}\right) d x \leq C_{1},
$$

where $C_{1}$ depends on $\|v\|_{\infty}$. Moreover, we obtain that for all $n \in \mathbb{N}$,

$$
\left\|\nabla u_{n}\right\| \leq C_{2}
$$

and

$$
\frac{1}{n} \int_{\Omega}\left|\nabla u_{n}\right|^{2} d x \leq C_{3}
$$

Thus, $\left\{u_{n}\right\}_{n \in \mathbb{N}}$ is bounded in $W^{1,1}(\Omega)$ and we may extract a subsequence such that $u_{n}$ converges in $L^{1}(\Omega)$. Now, by (4.15) and (4.16), we know that $u_{n} \rightarrow u$ in $L^{2}(\Omega)$ and $u \in$ $\operatorname{BV}(\Omega) \cap L^{2}(\Omega)$.

Observe that by (4.6) and (4.17), $A_{n}(x)\left(\nabla u_{n}\right)=A_{n}(\cdot)\left(\nabla u_{n}\right)$ is bounded in $L^{2}\left(\Omega ; \mathbb{R}^{N}\right)$ and weakly relatively compact in $L^{2}\left(\Omega ; \mathbb{R}^{N}\right)$. We may assume that

$$
A_{n}(\cdot)\left(\nabla u_{n}\right) \rightarrow z \quad \text { as } n \rightarrow \infty \text { weakly in } L^{2}\left(\Omega ; \mathbb{R}^{N}\right) .
$$

By (4.17) and $\frac{1}{n}\left|\nabla u_{n}\right| \rightarrow 0$ in $L^{2}(\Omega)$, we also have that

$$
A_{\frac{1}{n}}(\cdot)\left(\nabla u_{n}\right) \rightarrow z \quad \text { as } n \rightarrow \infty \text { weakly in } L^{2}\left(\Omega ; \mathbb{R}^{N}\right) .
$$

Given $\varphi \in C_{0}^{\infty}$ and taking $w=u_{n} \pm \varphi$ in (4.14), we have

$$
\int_{\Omega}\left(w-u_{n}\right) \varphi d x=\int_{\Omega} A_{n}(x)\left(\nabla u_{n}\right) \cdot \nabla \varphi d x
$$

and letting $n \rightarrow+\infty$, it follows that

$$
\int_{\Omega}(w-u) \varphi d x=\int_{\Omega} z \cdot \nabla \varphi d x
$$

that is, $v-u=-\operatorname{div}(z)$ in $\mathcal{D}^{\prime}(\Omega)$, and

$$
\operatorname{div}\left(A_{\frac{1}{n}}(\cdot)\left(\nabla u_{n}\right)\right) \rightarrow \operatorname{div}(z) \quad \text { as } n \rightarrow \infty \text { weakly in } L^{2}\left(\Omega ; \mathbb{R}^{N}\right) .
$$


From the proof of Proposition 4 in [11], we obtain that $f^{*}(x, z(x)) \leq 1$. Moreover, by (4.17) and (4.18), it implies that $z \in \mathcal{M}_{f}^{2}(\Omega)$. Now, we prove that $u, v$ and $z$ verify (4.13). Applying the Lebesgue convergence theorem in (4.14), there exists $u \in \operatorname{BV}(\Omega) \cap L^{2}(\Omega)$ for every $w \in W_{p}^{1,2}(\Omega) \cap L^{\infty}(\Omega)$,

$$
\begin{aligned}
\int_{\Omega}(w-u)(v-u) d x & \leq \int_{\Omega} z \cdot \nabla\left(w-u_{n}\right) d x \\
& \leq \int_{\Omega} z \cdot \nabla w d x-\int_{\Omega} z \cdot \nabla u d x \\
& =\int_{\Omega} z \cdot \nabla w d x-\int_{\Omega}(z, D u) \\
& =\int_{\Omega} z \cdot \nabla w d x-\int_{\Omega}|D u|_{f} .
\end{aligned}
$$

To prove (4.13), we assume that there exists $w_{0} \in W_{p}^{1,2}(\Omega) \cap L^{\infty}(\Omega)$. Let $w \in W^{1,1}(\Omega) \cap$ $L^{2}(\Omega)$ and let $w_{n} \in W_{p}^{1,2}(\Omega) \cap L^{\infty}(\Omega)$ be such that $w_{n} \rightarrow w$ in $L^{2}(\Omega)$ as $n \rightarrow \infty$. Using $w_{n}$ as a test function in (4.20) and letting $n \rightarrow \infty$, we obtain (4.13). That is, $(u, v-u) \in \mathcal{A}$.

Step 2. Now let us prove that $D(\mathcal{A})$ is dense in $L^{2}(\Omega)$. We only need to prove that $C_{0}^{\infty}(\Omega) \subseteq \overline{D(\mathcal{A})}^{L^{2}(\Omega)}$. Let $v \in C_{0}^{\infty}(\Omega)$. By Step 1 , we have that $v \in R\left(I+\frac{1}{n} \mathcal{A}\right)$ for all $n \in \mathbb{N}$. Thus, for each $n \in \mathbb{N}$, there exists $u_{n} \in D(\mathcal{A})$ such that $\left(u_{n}, v-u_{n}\right) \in \mathcal{A}$ and, in consequence, there exists some $z_{n} \in \mathcal{M}_{f}^{2}(\Omega)$ such that $n\left(v-u_{n}\right)=-\operatorname{div}\left(z_{n}\right)$ in $\mathcal{D}^{\prime}(\Omega)$ and

$$
n \int_{\Omega}\left(w-u_{n}\right)\left(v-u_{n}\right) d x \leq \int_{\Omega} z_{n} \cdot \nabla w d x-\int_{\Omega}|D u|_{f} \leq \int_{\Omega} z_{n} \cdot \nabla w d x \leq \int_{\Omega}|\nabla w|
$$

for every $w \in \operatorname{BV}(\Omega) \cap L^{2}(\Omega)$. Taking $w=v$ in the above inequality, we get

$$
\int_{\Omega}\left(v-u_{n}\right)^{2} d x \leq \frac{C}{n} \int_{\Omega}|\nabla v| d x
$$

Letting $n \rightarrow \infty$, it follows that $u_{n} \rightarrow v$ in $L^{2}(\Omega)$. This implies that $v \in \overline{D(\mathcal{A})}^{L^{2}(\Omega)}$.

Proof of Proposition 4.1 Let $p \in P_{0}$ and $(u, v),(\tilde{u}, \tilde{v}) \in \mathcal{A}$. Let $z, \tilde{z} \in \mathcal{M}_{f}^{2}(\Omega)$ be such that $v=-\operatorname{div}(z), \tilde{v}=-\operatorname{div}(\tilde{z})$ and

$$
\begin{aligned}
& \int_{\Omega}(w-u) v d x \leq \int_{\Omega}(z, D w)-\int_{\Omega}|D u|_{f}, \\
& \int_{\Omega}(w-\tilde{u}) \tilde{v} d x \leq \int_{\Omega}(\tilde{z}, D w)-\int_{\Omega}|D \tilde{u}|_{f}
\end{aligned}
$$

for every $w \in \mathrm{BV}(\Omega) \cap L^{2}(\Omega)$. Taking $w=u-p(u-\tilde{u})$ as a test function in (4.21), taking $w=\tilde{u}+p(u-\tilde{u})$ as a test function in (4.22), and by Theorem 2 in [4], we have that

$$
\begin{aligned}
\int_{\Omega} p(u-\tilde{u})(v-\tilde{v}) d x & \geq \int_{\Omega}(z-\tilde{z}, D p(u-\tilde{u})) \\
& =\int_{\Omega} \theta(z-\tilde{z}, D p(u-\tilde{u}), x)|D p(u-\tilde{u})| \geq 0 .
\end{aligned}
$$

We get the operator $\mathcal{A}$ is completely accretive in $L^{2}(\Omega)$. 
Now, we prove that $\mathcal{A}$ is closed. Let $\left(u_{n}, v_{n}\right) \in \mathcal{A}$ such that $\left(u_{n}, v_{n}\right) \rightarrow(u, v)$ in $L^{2}(\Omega) \times$ $L^{2}(\Omega)$. Since $\left(u_{n}, v_{n}\right) \in \mathcal{A}$, there exists $z_{n} \in \mathcal{M}_{f}^{2}(\Omega)$ with $v_{n}=-\operatorname{div}\left(z_{n}\right)$ in $\mathcal{D}^{\prime}(\Omega)$ such that

$$
\int_{\Omega}\left(w-u_{n}\right) v_{n} d x \leq \int_{\Omega}\left(z_{n}, D w\right)-\int_{\Omega}\left|D u_{n}\right|_{f}
$$

for every $w \in \operatorname{BV}(\Omega) \cap L^{2}(\Omega)$. Since $\left\|z_{n}\right\|_{\infty} \leq C$, we may assume that

$$
z_{n} \rightarrow z \quad \text { in the weak" topology of } L^{\infty}\left(\Omega ; \mathbb{R}^{N}\right) .
$$

Working as before, it is easy to see that $z \in \mathcal{M}_{f}^{2}(\Omega)$. Moreover, since $v_{n} \rightarrow v$ in $L^{2}(\Omega)$, we have $v=-\operatorname{div}(z)$ in $\mathcal{D}^{\prime}(\Omega)$, and

$$
\lim _{n \rightarrow \infty} \int_{\Omega}\left(z_{n}, D w\right)=\int_{\Omega}(z, D w)
$$

Letting $n \rightarrow \infty$ in (4.23), and having in mind the lower semicontinuity of the functional $\mathcal{F}$ defined in (2.13), we obtain that

$$
\int_{\Omega}(w-u) v d x \leq \int_{\Omega}(z, D w)-\int_{\Omega}|D u|_{f}
$$

Consequently, $(u, v) \in \mathcal{A}$. By Lemma 4.5 , it follows that $\mathcal{A}$ is $m$-completely accretive in $L^{2}(\Omega)$. By Crandall-Liggett's theorem, $\mathcal{A}$ generates a contraction semigroup in $L^{2}(\Omega)$ given by the exponential formula

$$
e^{-t \mathcal{A}} u_{0}=\lim _{n \rightarrow \infty}\left(I+\frac{t}{n} \mathcal{A}\right)^{-n} u_{0} \quad \text { for any } u_{0} \in L^{2}(\Omega) .
$$

The function $u(t)=e^{-t \mathcal{A}} u_{0}$ is a mild solution of

$$
\frac{d u}{d t}+\mathcal{A} u \ni 0
$$

with $u(0)=u_{0}$.

Proof of Proposition 4.2 We first prove that $\mathcal{A} \subseteq \partial \mathcal{F}$. Let $(u, v) \in \mathcal{A}$ and $w \in \operatorname{BV}(\Omega) \cap L^{2}(\Omega)$, there exists $z \in \mathcal{M}_{f}^{2}(\Omega)$ with $v=-\operatorname{div}(z)$ in $\mathcal{D}^{\prime}(\Omega)$ such that

$$
\int_{\Omega}(w-u) v d x \leq \int_{\Omega}(z, D w)-\int_{\Omega}|D u|_{f} \leq \mathcal{F}(w)-\mathcal{F}(u)
$$

for every $w \in \mathrm{BV}(\Omega) \cap L^{2}(\Omega)$. Thus, $(u, v) \in \partial \mathcal{F}$, that is, $\mathcal{A} \subseteq \partial \mathcal{F}$.

Next, by the proof of Proposition 4.1, we have that the operator $\mathcal{A}$ is closed. Since $\mathcal{A} \subseteq$ $\partial \mathcal{F}$ and $L^{\infty}(\Omega) \subset R(I+\mathcal{A})$, we have that $\partial \mathcal{F}=\overline{\mathcal{A}}^{L^{2}(\Omega) \times L^{2}(\Omega)}$.

Proof of Theorem 3.3 As a consequence of Proposition 4.2, the semigroups generated by $\mathcal{A}$ and by $\partial \mathcal{F}$ coincide, and therefore $u(t, x):=e^{-t \mathcal{A}} u_{0}(x)$ is a strong solution of

$$
\frac{d u(t)}{d t}+\mathcal{A} u(t) \ni 0, \quad \text { in } L^{2}(\Omega), t \in[0, T]
$$


with $u(0)=u_{0}$, i.e., $u \in W_{\mathrm{loc}}^{1,2}\left([0, T] ; L^{2}(\Omega)\right)$ and $\left(u(t), u^{\prime}(t)\right) \in \mathcal{A}$ for a.e. $t \in[0, T]$. Then we have $u^{\prime}(t)=\operatorname{div}(z(t))$ in $\mathcal{D}^{\prime}([0, T] \times \Omega)$. By the characterization (i) in Lemma 4.3, we have (3.2) and (3.3) hold. The contractivity estimate (3.5) follows directly from the nonlinear semigroup theory.

Let us define several operators that will be needed in this section. The single-valued operator $B_{h}$ is defined in $L^{2}(\Omega)$ as

$$
B_{h}:=\left\{(u, v) \in L^{2}(\Omega) \times L^{2}(\Omega): v=h(\cdot, u(\cdot)) \text { a.e. }\right\} .
$$

Take $H: L^{2}(\Omega) \rightarrow[0, T]$ defined by

$$
H(u):= \begin{cases}\int_{\Omega} k(x, u(x)) d x, & x \rightarrow k(x, u(x)) \in L^{1}(\Omega), \\ +\infty, & \text { otherwise }\end{cases}
$$

where $k(x, u(x)):=\int_{0}^{r} h(x, s) d s$. It is easy to see that $L^{\infty}(\Omega) \subset \mathcal{D}(H)$ and $H$ is convex. Moreover, by Fatou's lemma, $H$ is lower semicontinuous. Hence, $\partial H$ is a maximal monotone graph in $L^{2}(\Omega)$.

Thus, to prove Theorem 3.4, we only need to obtain the following result.

Lemma 4.6 $\partial(H+\mathcal{F})=\mathcal{A}+B_{h}$.

Proof From $(u, v) \in \mathcal{A}+B_{h}$, we have $u \in \operatorname{BV}(\Omega) \cap L^{2}(\Omega), v \in L^{2}(\Omega)$ and there exists $z \in$ $\mathcal{M}_{f}^{2}(\Omega), v=-\operatorname{div}(z)+h(x, u)$ in $\mathcal{D}^{\prime}(\Omega)$ such that

$$
\int_{\Omega}(w-u) v d x \leq \int_{\Omega}(z, D w) d x-\int_{\Omega}|D u|_{f}+\int_{\Omega}(w-u) h(x, u) d x
$$

for all $w \in W^{1,1}(\Omega) \cap L^{2}(\Omega)$. Since $h(x, u)$ satisfies $\left(\mathrm{M}_{1}\right)$, there exists $z(x) \in[u(x), w(x)]$ such that

$$
\int_{\Omega} \int_{u}^{v} h(x, s) d s d x=\int_{\Omega}(w-u) h(x, z(x)) d x \geq \int_{\Omega}(w-u) h(x, u) d x .
$$

By the above inequality and $\mathcal{F}$ being lower semicontinuous in (4.25), we have that

$$
\begin{aligned}
\int_{\Omega}(w-u) v d x & \leq \int_{\Omega}(z, D w) d x-\int_{\Omega}|D u|_{f}+\int_{\Omega}(w-u) h(x, u) d x \\
& \leq \int_{\Omega}|D w|_{f}-\int_{\Omega}|D u|_{f}+\int_{\Omega} \int_{u(x)}^{v(x)} h(x, s) d s d x \\
& =(H+\mathcal{F})(w)-(H+\mathcal{F})(u) .
\end{aligned}
$$

We have proved that $\mathcal{A}+B_{h} \subseteq \partial(H+\mathcal{F})$.

By Proposition 14 in [19], we have that $L^{\infty}(\Omega) \subseteq R\left(I+\mathcal{A}+B_{h}\right)$, and the operator $\mathcal{A}+B_{h}$ is closed. Hence, $\partial(H+\mathcal{F})={\overline{\mathcal{A}+B_{h}}}^{L^{2}(\Omega) \times L^{2}(\Omega)}$.

Using Crandall-Liggett's theorem and a similar proof of Theorem 3.3 again, we obtain that Theorem 3.4 holds. 
Competing interests

The authors declare that they have no competing interests.

\section{Authors' contributions}

All authors contributed equally to the manuscript and typed, read and approved the final manuscript.

\section{Author details}

${ }^{1}$ School of Mathematics, Shandong University, Jinan, Shandong 250100, P.R. China. ${ }^{2}$ College of Science, China University of Petroleum (East China), Qingdao, Shandong 266555, P.R. China.

\section{Acknowledgements}

We would like to thank the anonymous referees for their constructive comments, which were very helpful for improving this paper. The authors acknowledge the financial support of this research by the National Natural Science Foundation of China (Grant No. 10871117), NSFSP (Grant No. ZR2010AM013) and Fundamental Research Funds for the Central Universities (12CX04081A, 11CX04058A)

\section{Received: 7 December 2012 Accepted: 30 April 2013 Published: 18 June 2013}

\section{References}

1. Giga, MH, Giga, Y, Kobayashi, R: Very singular diffusion equations. In: Proc. of Taniguchi Conf. on Math. Advanced Studies in Pure Mathematics, vol. 31, pp. 93-125 (2003)

2. Rudin, L, Osher, S: Total variation based image restoration with free local constraints. In: Proc. of the IEEE ICIP-94, vol. 1 , pp. 31-35 (1994)

3. Rudin, L, Osher, S, Fatemi, E: Nonlinear total variation based noise removal algorithms. Physica D 60, $259-268$ (1992)

4. Andreu, F, Ballester, C, Caselles, V, Mazón, JM: Minimizing total variation flow. Differ. Integral Equ. 4(3), 321-360 (2001)

5. Bellettini, G, Caselles, V, Novaga, M: The total variation flow in $\mathbb{R}^{N}$. J. Differ. Equ. 184, 475-525 (2002)

6. Andreu, F, Ballester, C, Caselles, V, Mazón, JM: The Dirichlet problem for the total variation flow. J. Funct. Anal. 180(2) 347-403 (2001)

7. Andreu, F, Caselles, V, Mazón, JM: A strongly degenerate quasilinear equation: the parabolic case. Arch. Ration. Mech. Anal. 176, 415-453 (2005)

8. Alvarez, L, Lions, PL, Morel, JM: Image selective smoothing and edge detection by nonlinear diffusion. SIAM J. Numer. Anal. 29, 845-866 (1992)

9. Andreu, F, Caselles, V, Mazón, JM: A strongly degenerate quasilinear equations: the elliptic case. Ann. Sc. Norm. Super. Pisa, Cl. Sci. 111, 555-587 (2004)

10. Chambolle, A: An algorithm for mean curvature motion. Interfaces Free Bound. 6, 195-218 (2004)

11. Moll, JS: The anisotropic total variation flow. Math. Ann. 332, 177-218 (2005)

12. Lin, FH, Yang, XP: Geometric Measure Theory. International Press, New York (2002)

13. Anzellotti, G: Pairings between measures and bounded functions and compensated compactness. Ann. Mat. Pura Appl. 135, 293-318 (1993)

14. Amar, M, Belletini, G: A notion of total variation depending on metric with discontinuous coefficients. Ann. Inst. Henri Poincaré 11, 91-133 (1994)

15. Benilan, P, Crandall, MG: Completely accretive operators. In: Clement, P, Mitidieri, E, de Pagter, B (eds.) Semigroups Theory and Evolution Equations. Dekker, New York (1991)

16. Crandall, MG, Liggett, TM: Generation of semigroups of nonlinear transformations on general Banach spaces. Am. J. Math. 93, 265-298 (1971)

17. Brezis, H: Operateurs Maximaux Monotones. North-Holland, Amsterdam (1973)

18. Lera, J, Lions, JL: Quelques résultats de Visik sur le problèmes elliptiques non linéaires par le méthodes de Minity-Browder. Bull. Soc. Math. Fr. 93, 97-107 (1965)

19. García-Falset, J: The asymptotic behavior of the solutions of the Cauchy problem generated by $\phi$-accretive operators J. Math. Anal. Appl. 310, 594-608 (2005)

doi:10.1186/1687-2770-2013-147

Cite this article as: Rui and Si: An anisotropic quasilinear problem with perturbations. Boundary Value Problems 2013 2013:147.

\section{Submit your manuscript to a SpringerOpen ${ }^{\circ}$ journal and benefit from:}

- Convenient online submission

Rigorous peer review

- Immediate publication on acceptance

- Open access: articles freely available online

- High visibility within the field

- Retaining the copyright to your article 